\title{
A Firework-Based GMPPT with Variable Sampling Time for PV Systems
}

\author{
N. Khalessi, ${ }^{1}$ M. Niroomand $\left(10,{ }^{2}\right.$ J. Dadkhah, ${ }^{2}$ and S. Y. Nikouei ${ }^{2}$ \\ ${ }^{1}$ Electrical Engineering Department, Islamic Azad University of Najafabad, Najafabad, Isfahan, Iran \\ ${ }^{2}$ Department of Electrical Engineering, University of Isfahan, Isfahan, Isfahan, Iran \\ Correspondence should be addressed to M. Niroomand; mehdi_niroomand@yahoo.com
}

Received 13 August 2020; Revised 25 October 2020; Accepted 30 October 2020; Published 18 November 2020

Academic Editor: Antonino Laudani

Copyright (c) 2020 N. Khalessi et al. This is an open access article distributed under the Creative Commons Attribution License, which permits unrestricted use, distribution, and reproduction in any medium, provided the original work is properly cited.

Photovoltaic systems have a nonlinear characteristic in which there is one optimum operating point called Maximum Power Point (MPP). However, when PV panels are partially shaded by surrounding objects, there are several MPPs, of which one of them is Global MPP (GMPP). Therefore, conventional Maximum Power Point Tracking (MPPT) algorithms get trapped into local MPPs. As a result, a multitude of Global MPPT (GMPPT) algorithms have been proposed. An outstanding GMPPT algorithm as well as the fast-tracking speed should find GMPP in complicated shading patterns where not only there are lots of MPPs, but also the peaks are close together. Therefore, in this paper, a novel GMPPT based on firework algorithm is proposed which is able to find GMPP in complicated shading patterns with fast tracking speed. Moreover, the firework is combined with Perturb-and-Observe $(\mathrm{P} \& \mathrm{O})$ algorithm to reduce the computational effort in a way that the firework is only used to recognize GMPP; afterwards, $\mathrm{P} \& \mathrm{O}$ algorithm completes the tracking. Furthermore, the variable sampling time technique, based on the system settling time, speeds up the tracking process considerably. Finally, the proposed method is compared with previous works, simulated, and implemented on an experimental setup to prove its superiority.

\section{Introduction}

Renewable energy is an attractive alternative to fossil fuels because of its environmentally friendly manner. Photovoltaic (PV) systems stand out from other kinds of renewable energy since they can be used in a multitude of applications, either on a small scale or large one, such as water pumping, air conditioning, lighting, and electrical vehicle [1]. However, nonlinear characteristic of PV systems, including a maximum power point (MPP), needs special measurements to extract maximum power from $\mathrm{PV}$ panels. Furthermore, when PV panels are partially shaded, instead of an MPP, there are multiple MPPs. Therefore, conventional Maximum Power Point Tracking (MPPT) algorithms such as Incremental conductance (INC), Perturb and Observe (P\&O), and Ripple Current Control (RCC) [2-6] get trapped into local MPPs when Partial Shading condition (PSC) occurs. In fact, these methods are effective in uniform irradiance.
As a result, Global MPPT (GMPPT) is developed to differentiate the local MPPs from Global MPP (GMPP). An ideal GMPPT algorithm should have fast tracking speed so that in variable weather condition it does not fail to track GMPP. In addition, GMPPT algorithm must have good ability to recognize GMPP in complicated shading patterns which mainly happens in large-scale PV systems where there are multiple PV strings in parallel. Finally, the computational effort should be minimized to reduce the implementation cost. In the literature, intelligent methods such as fuzzy logic [7] and artificial neural network (ANN) [8] are proposed as GMPPT. However, the fuzzy logic method is computationally heavy, and ANN requires data sets for the training stage. Furthermore, the fuzzy logic and ANN are fused together to increase the robustness of GMPPT in different configurations of PV panels [9].

Another kind of GMPPT consists of two stages in which the limit of local MPP is identified firstly, and consequently a conventional algorithm is run to find the 
exact position of MPP. Finally, all the identified MPPs are compared to find GMPP; therefore, the knowledge of all MPPs is needed $[10,11]$. As a result, to reduce the complexity, the load line is used to directly place the operating point to the vicinity of GMPP; however, this method fails to find GMPP in the left side of the load line $[12,13]$.

Line searching algorithm is used to measure the PV power in two operating points, and consequently the step size of GMPPT is determined $[14,15]$. This method is similar to the variable step size $\mathrm{P} \& \mathrm{O}$ while the step size, here, is determined by Fibonacci [14] and dividing rectangles [15]. Nevertheless, the methods fail to track GMPP in complicated shading patterns. Finally, some of the methods are system dependent in which P-V curve should first be evaluated [16].

Particle Swarm Optimization (PSO), either combined with perturb-and-observe-based MPPT [17] or alone [18], is used to perform GMPPT. Generally, its performance has been satisfying; however, PSO is computationally heavy, especially, when undergoing modifications. Moreover, there are some parameters that must be tuned in the design stage. Therefore, its performance is system dependent.

A novel swarm intelligence method called firework algorithm (FWA) is recently proposed which is inspired from the fireworks in the sky [19-22]. This algorithm has been demonstrated to be superior to the PSO algorithm in terms of optimization accuracy and convergence time. With a desirable mix of exploitation and exploration, the FWA is quite suitable for PV GMPP tracking, as the GMPP tracker needs to avoid getting trapped in local maximum power points and accurately determine the GMPP as well.

In this paper, FWA is applied to find GMPP and fused with $\mathrm{P} \& \mathrm{O}$ algorithm to reduce the computational effort in the steady state. In fact, GMPP is first identified by FWA, and consequently $\mathrm{P} \& \mathrm{O}$ algorithm is used to track the fluctuation of the PV power in response to irradiance and temperature variations. Therefore, FWA which is computationally heavier than $\mathrm{P} \& \mathrm{O}$ algorithm is only used when PSC occurs, and the PV system is mainly based on the simple $\mathrm{P} \& \mathrm{O}$ algorithm. Furthermore, variable sampling time technique is used to speed up the transient time. Indeed, the PV system is modelled by its space-state equations, and the voltage settling time is investigated by the model. Afterwards, based on the perturbation amplitude $(\Delta D)$, a proper sampling time, which is equal to the system settling at certain $\Delta D$, is allocated to FWA. Therefore, FWA waits neither too long so that the tracking speed reduces nor too short so that the PV voltage and current are sampled in the transient state. Thus, the proposed method greatly satisfies the criteria of an ideal GMPPT.

The PV panel model and PV system behaviour in PSC is described in Section 2. The FWA is introduced in Section 3. The proposed method is presented in Section 4. Finally, the simulation and experimental results are presented in Sections 5 and 6 , respectively.

\section{PV Panel Characteristics}

To design a GMPPT algorithm which satisfies the ideal criterion, it is first essential to perceive how PV panel is modelled and how PSC affects the PV panel behaviour.

2.1. PV Panel Modelling. The nonlinear behaviour of the PV panel is evident in I-V curve where MPP represents the best operating point. A single model diode is used to produce the same behaviour of the PV panel in simulation. According to Figure 1, the diode takes into account the $p-n$ junction of the cell, the current source represents the photo-induced current, and $R_{\mathrm{S}}$ describes the internal cell resistances and contacts resistances. Therefore, the PV current is obtained from the following equation [23]:

$$
I_{\mathrm{PV}}=I_{\mathrm{g}}-I_{\mathrm{sat}}\left(\exp \left(\frac{q\left(V_{\mathrm{PV}}+I_{\mathrm{PV}} \cdot R_{\mathrm{S}}\right)}{n k T}\right)-1\right),
$$

where $n$ is the ideality factor, $k$ is the Boltzmann constant, $q$ is the electron charge, $T$ is the cell temperature, and $I_{\text {sat }}$ is the saturation current.

2.2. PV Panel Behaviour in PSC. A PV cell output voltage is quite low, so to reach a desired level of output voltage, they are connected in series. However, if a part of a string constituting of cells in series is shaded by surrounding objects, the current of the whole string is limited by the current of the shaded cell, and the extra current of the unshaded cells is dissipated in the resistor $R_{\mathrm{S}}$ and creates hotspots. Therefore, the bypass diodes are placed in parallel to several cells to bypass shaded cells and avoid creating hotspots (Figure 2(a)). Nevertheless, the bypass diodes protection makes the nonlinear behaviour of the PV system more complex since there are multiple peaks in PSC. Depending on the number of shaded cells, different $\mathrm{P}-\mathrm{V}$ curves are generated by each string. Afterwards, multipeak $\mathrm{P}-\mathrm{V}$ curves are combined because of the parallel connection, which increases the overall complexity (Figure 2(b)).

\section{Firework Algorithm}

Finding extremum points of the nonlinear functions is the aim of many optimization problems such as GMPPT. Moreover, a good solution as well as fast convergence rate should maintain high accuracy with minimum computational effort. The FWA which is inspired from explosion of fireworks in the sky is an interesting solution which has the mentioned features. It is able to solve nonlinear problems and complex numerical calculations in different field of optimization problems with high accuracy and convergence rate. Since PV systems in PSC have complex nonlinear behaviour, FWA can be used to find GMPP not only in complicated shading patterns but also with the fast tracking speed.

When a firework is set off, a surge of sparks fill the space. Therefore, according to Figure 3, the space can be treated as a search space in which the sparks, surrounding the origin of the firework, are possible solutions. 


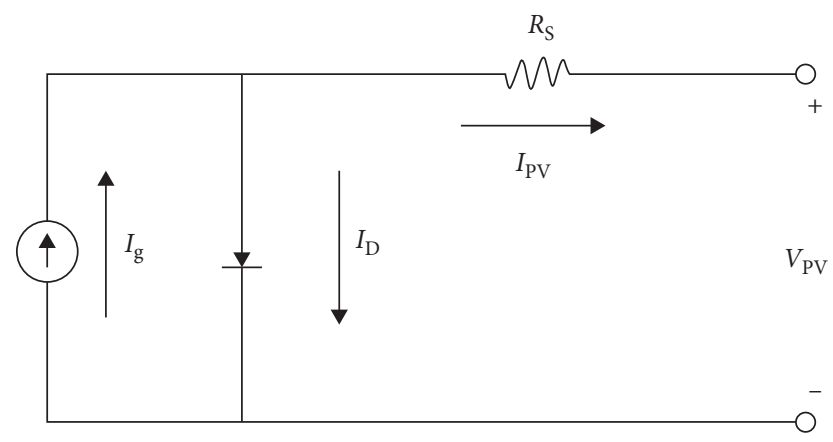

Figure 1: The single-diode model of a solar cell [23].
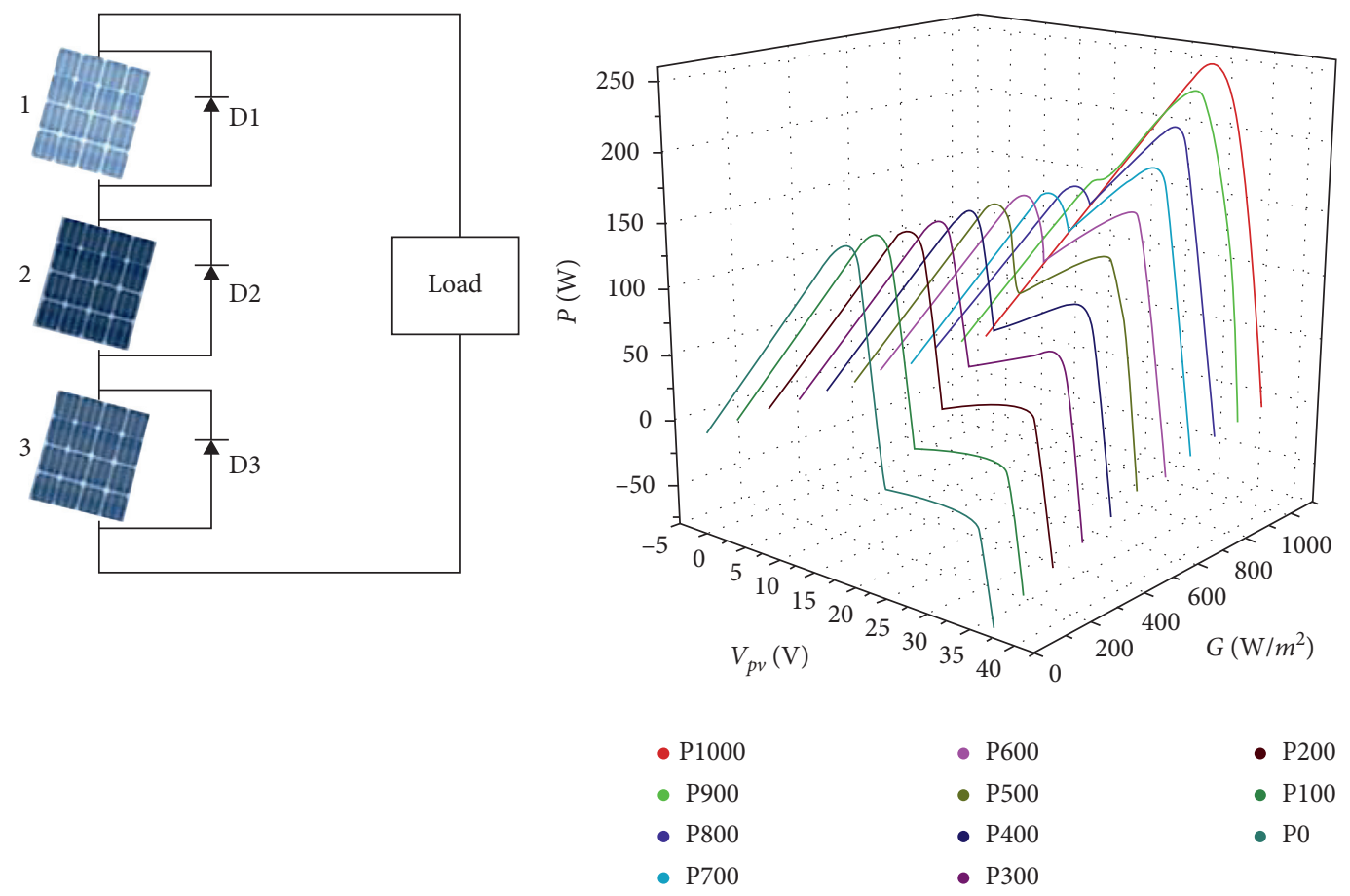

(a)

(b)

Figure 2: (a) Demonstration of bypass diodes. (b) P-V curve under PSC [24].

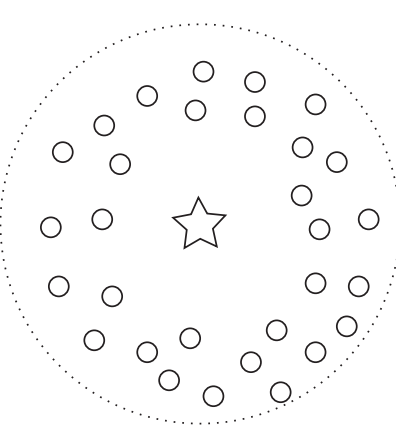

(a)

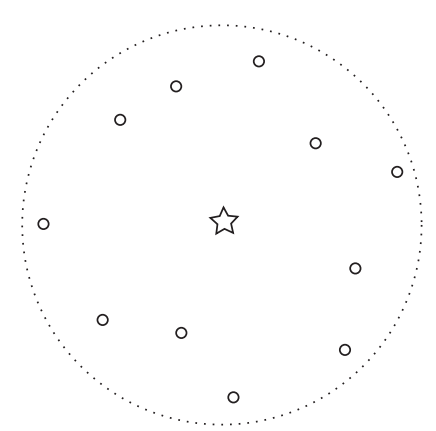

(b)

Figure 3: The firework explosion. (a) Good explosion. (b) Bad explosion [21]. 
The FWA finds the global minimum point of nonlinear functions. For instance, if a point $x_{j}$ is desirable to satisfy $f\left(x_{j}\right)=y$, FWA can be continuously set off in potential locations in the search space until a spark happens either at $x_{j}$ or close to it. In FWA, initially $n$ fireworks are exploded, and the fitness value of each of them is evaluated. If the fitness value is satisfactory, the successive fireworks are created with more dense sparks (Figure 3(a)). Otherwise, the search space is expanded by increasing the radius of sparks (Figure 3(b)). Therefore, this method ensures exploring the whole search space with high precision and the lowest computational effort. Moreover, in nonlinear optimization problems like GMPPT where local and global MPPs may be close together, FWA by creating different sparks can successfully find GMPP with fast convergence rate [19-22].

\section{The Proposed Method}

The proposed method is composed of FWA and P\&O algorithms. The former finds GMPP, and the latter tracks GMPP when irradiance and temperature change. According to Figure 4, FWA chooses $\mathbf{n}$ locations of the duty cycle as the origin of the fireworks, and consequently $\mathbf{n}$ other locations around the origins are selected to create sparks of the fireworks. Afterwards, the perturbation amplitude $(\Delta \mathbf{D})$ is calculated, and two different sampling times are assigned to FWA to allow the PV voltage and current settle in an acceptable range prior to sampling them. Then, the duty cycle vector is applied to the power converter, and the PV power is measured after proper sampling time. In each firework explosion, the maximum $\mathrm{PV}$ power and its corresponding duty cycle are saved in variables $\mathbf{P}_{\text {best }}$ and $\mathbf{D}_{\text {best }}$, respectively. Afterwards, if the current $\mathbf{P}_{\text {best }}$ is greater than $\mathbf{P}_{\mathbf{G}_{-} \text {best }}$, the stopping criterion is evaluated. Otherwise, a new firework is set off. To increase the accuracy of the proposed method, the stopping criterion should be satisfied four times. As soon as the stopping criterion is met, $\mathrm{P} \& \mathrm{O}$ algorithm is run. In the following, a detailed explanation of FWA and variable sampling time technique is provided.

4.1. PV Panel Modelling. In PV systems, the location of fireworks is the duty cycle of the power electronic converter. Therefore, the objective function (2) is formed where $\mathbf{P}\left(\mathbf{d}_{\mathbf{i}}\right)$ is the amount of the PV power at the duty cycle $\mathbf{d}_{\mathbf{i}}$.

$$
\mathbf{f}\left(\mathbf{d}_{\mathbf{i}}\right)=-\mathbf{P}\left(\mathbf{d}_{\mathbf{i}}\right) .
$$

The number of sparks is obtained by

$$
\mathbf{s}_{\mathbf{i}}=\mathbf{m} \cdot \frac{\mathbf{y}_{\max }-\mathbf{f}\left(\mathbf{x}_{\mathbf{i}}\right)+\xi}{\sum_{\mathbf{i}=1}^{\mathbf{n}}\left(\mathbf{y}_{\max }-\mathbf{f}\left(\mathbf{x}_{\mathbf{i}}\right)\right)+\boldsymbol{\xi}},
$$

where the total number of sparks is determined by $\mathbf{m}, \mathbf{y}_{\max }$ is the maximum amount of the objective function, and $\xi$ is a small positive value to avoid division by zero. Moreover, the number of sparks is limited by (4) since a large number of sparks not only increases the computational effort but also slows down the tracking speed.

$$
s_{i}= \begin{cases}\operatorname{round}(\mathbf{a} . m), & \text { if } s_{i}<\mathbf{a m}, \\ \operatorname{round}(\mathbf{b} . m), & \text { if } s_{\mathbf{i}}>\mathbf{b m}, \mathbf{a}<\mathbf{b}<1, \\ \operatorname{round}\left(s_{i}\right), & \text { otherwise, }\end{cases}
$$

where $\mathbf{a}$ and $\mathbf{b}$ are the constant values.

Consequently, the amplitude of sparks from the origin of explosion is given by

$$
\mathbf{A}_{\mathbf{i}}=\mathbf{A} \cdot \frac{\mathbf{f}\left(\mathbf{x}_{\mathbf{i}}\right)-\mathbf{y}_{\min }+\xi}{\sum_{\mathbf{i}=1}^{\mathbf{n}}\left(\mathbf{f}\left(\mathbf{x}_{\mathbf{i}}\right)-\mathbf{y}_{\min }\right)+\xi}
$$

where A determines the largest amplitude of the sparks, and $\mathbf{y}_{\min }$ is the minimum value of the objective function.

4.2. Variable Sampling Time Techniques. The GMPPT algorithm applies the perturbation amplitude $\Delta \mathbf{D}$ to the duty cycle of the DC-DC power converter. Since the PV voltage and current should be sampled in the steady state, GMPPT algorithm has to wait enough prior to applying next perturbation so that the transient time is passed, and the data are sampled. As a result, a proper sampling time $\left(\mathbf{T}_{\mathrm{s}}\right)$ must be allocated to GMPPT which is equal to the system settling time. However, a fixed sampling time cannot be valid for different values of $\Delta \mathbf{D}$ because the bigger $\Delta \mathbf{D}$ is, the longer settling time is.

Therefore, the variable sampling time is proposed to describe the dynamic behaviour of PV system based on the amplitude of $\Delta \mathbf{D}$.

Thus, the PV system model is first investigated to analyze the step response of the PV voltage to different values of $\Delta \mathbf{D}$ where the settling times can be found. The PV system constituting of the boost converter and a resistive load is described by (6) where $\mathbf{i}_{\mathbf{L}}$ (current of the inductor), $\mathbf{v}_{\mathbf{C} 1}$ (voltage of the capacitor parallel with the PV panel) and $\mathbf{v}_{\mathrm{C} 2}$ (voltage of the capacitor parallel with the load) are state variables.

$$
\left[\begin{array}{c}
\frac{\mathbf{d i}_{\mathbf{L}}}{\mathbf{d t}} \\
\frac{\mathbf{d v}_{\mathrm{C} 1}}{\mathrm{~d} t} \\
\frac{\mathrm{d} v_{C 2}}{\mathrm{~d} t}
\end{array}\right]=\left[\begin{array}{ccc}
0 & \frac{-1}{C_{1}} & 0 \\
\frac{1}{L} & 0 & \frac{-(1-D)}{\mathrm{L}} \\
0 & \frac{1-D}{C_{2}} & \frac{-1}{R C_{2}}
\end{array}\right]\left[\begin{array}{c}
i_{L} \\
v_{C 1} \\
v_{C 2}
\end{array}\right]+\left[\begin{array}{c}
\frac{1}{C_{1}} \\
0 \\
0
\end{array}\right] I_{\mathrm{in}} .
$$

The PV current is considered constant in a specific operating point to linearize the model. Therefore, the PV power and voltage settling times are alike. Hence, the step responses of the PV voltage to different values of $\Delta D$ are depicted in Figure 5 by (6) and (7).

$$
y(t)=C x(t)+D I_{\text {in }}
$$

where $C=\left[\begin{array}{lll}0 & 1 & 0\end{array}\right]$ and $D=0$.

Based on Figure 5, it is evident that the sampling time equal to $4 \mathrm{~ms}$ can represent acceptable delay time for $\Delta D$ smaller than $1.5 \%$. On the contrary, sampling time equal to 


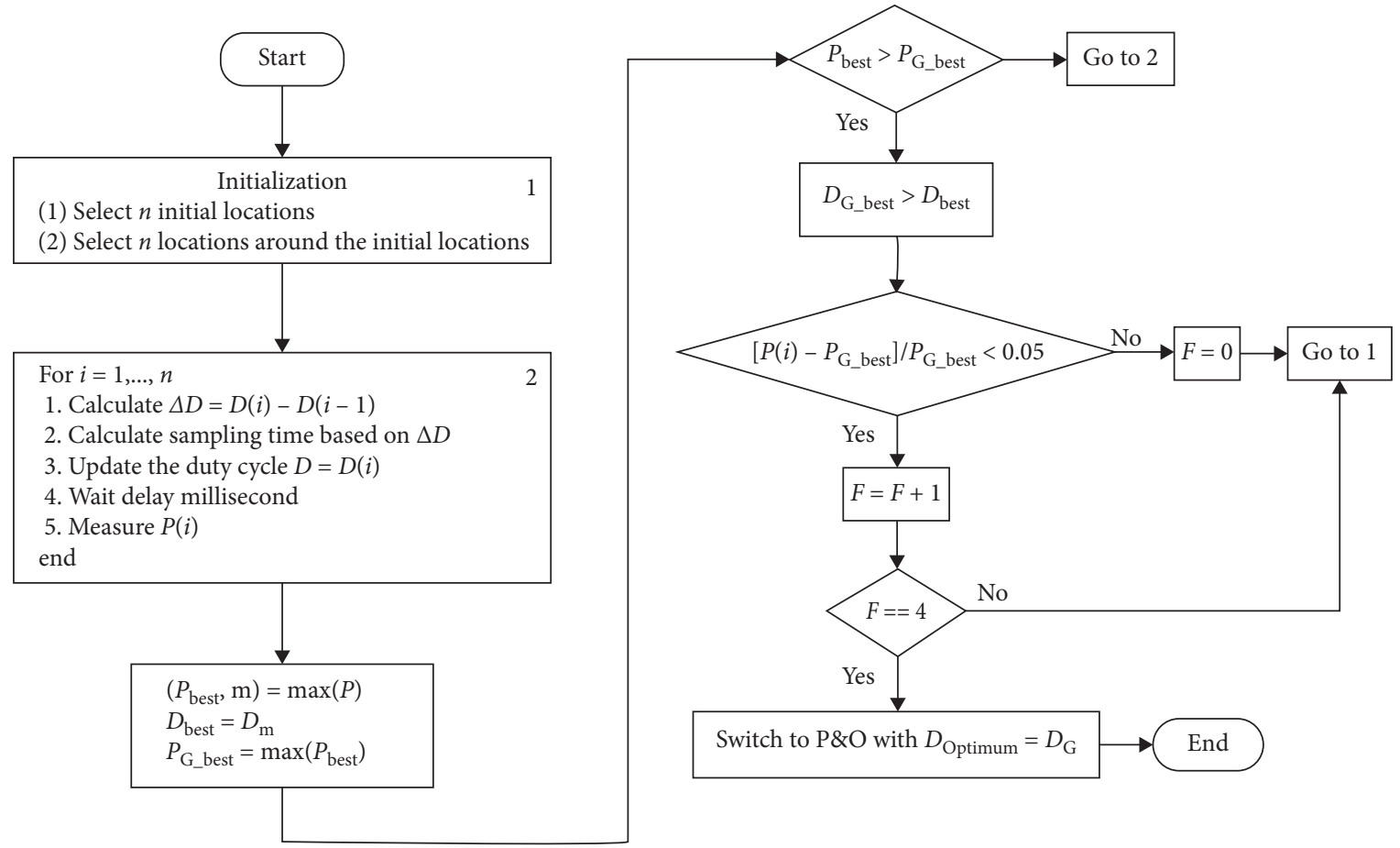

FIGURE 4: Flowchart of the proposed method.

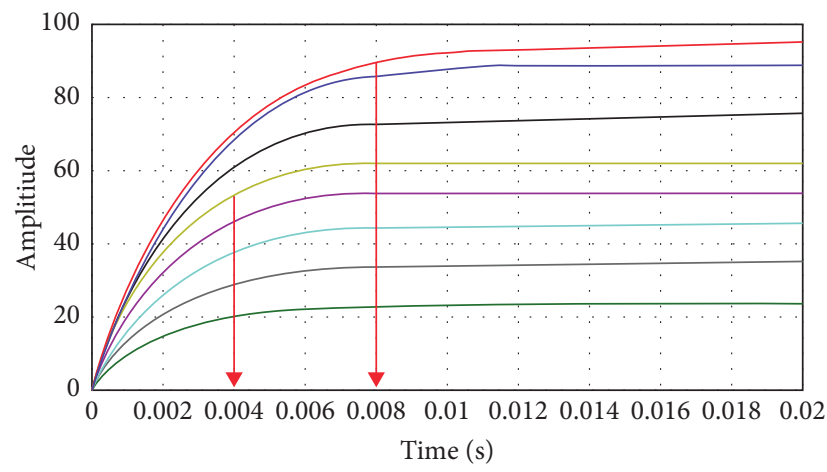

$$
\begin{aligned}
& -\Delta D=80 \% \quad-\triangle D=20 \% \\
& -\Delta D=60 \%-\Delta D=16 \% \\
& -\Delta D=40 \%-\Delta D=12 \% \\
& \Delta D=27 \% \quad-\Delta D=4 \%
\end{aligned}
$$

Figure 5: The step responses of the PV voltage to different values of $\Delta D$.

$8 \mathrm{~ms}$ can represent acceptable delay time for $\Delta D$ bigger than $1.5 \%$. Thus, the variable sampling time is selected by

$$
\begin{cases}\Delta D \leq 1.5, & T_{\mathrm{s}}=0.004 \mathrm{~s} \\ \Delta D>1.5, & T_{\mathrm{s}}=0.008 \mathrm{~s}\end{cases}
$$

\section{Simulation Results}

The proposed method is simulated in different conditions to evaluate its robustness and the tracking speed. The simulations are conducted by the link of PSIM and MAT$\mathrm{LAB} /$ Simulink software to increase the accuracy of simulations.
5.1. Simulation. In order to test and validate the proposed method, the PV setup constitutes of the boost converter (Figure 6). To show the severity of partial shading conditions, two different shades having three peaks and four peaks in the P-V curve are considered $4 \mathrm{~S}$ and $4 \mathrm{~S}-3 \mathrm{P}$ configurations.

The proposed method is simulated in four different shading patterns with fixed and variable sampling times (Figure 7).

According to Figure 8, the proposed method successfully finds GMPP in all of the patterns, and also the variable sampling time leads to faster tracking speed compared with the fixed one in a way that the tracking speed improves by approximately $50 \%$. When the stopping criterion of FWA is 


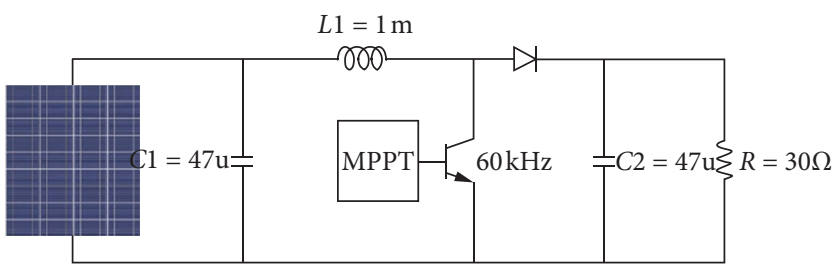

FIgure 6: The PV setup used in the simulation.

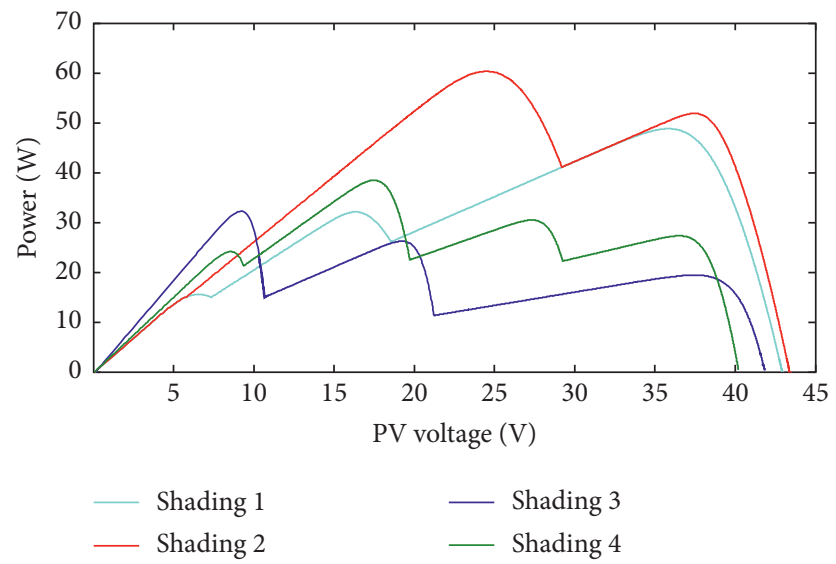

FIgURE 7: The shading patterns used in the simulations.
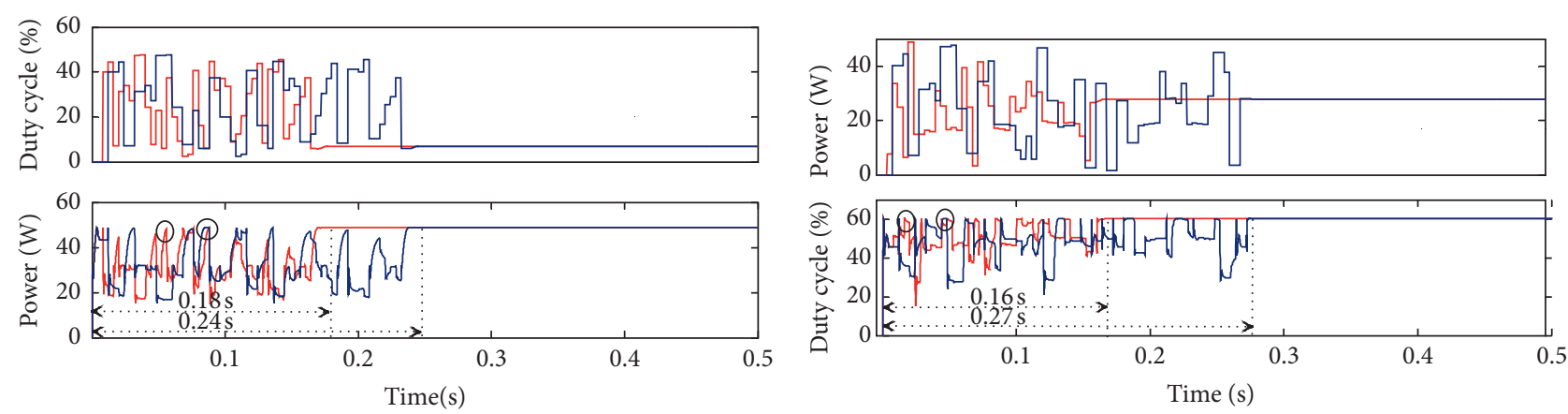

_ Variable Ts

_ Variable Ts

— Fixed Ts

— Fixed $T \mathrm{~s}$

(a)
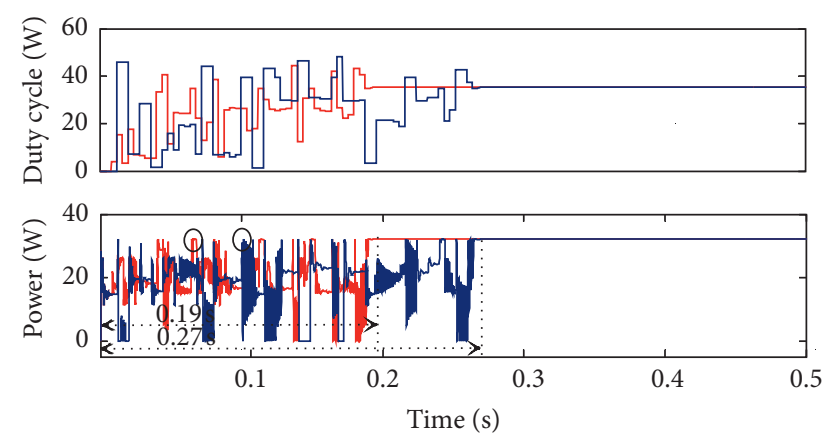

— Variable Ts

_ Fixed Ts
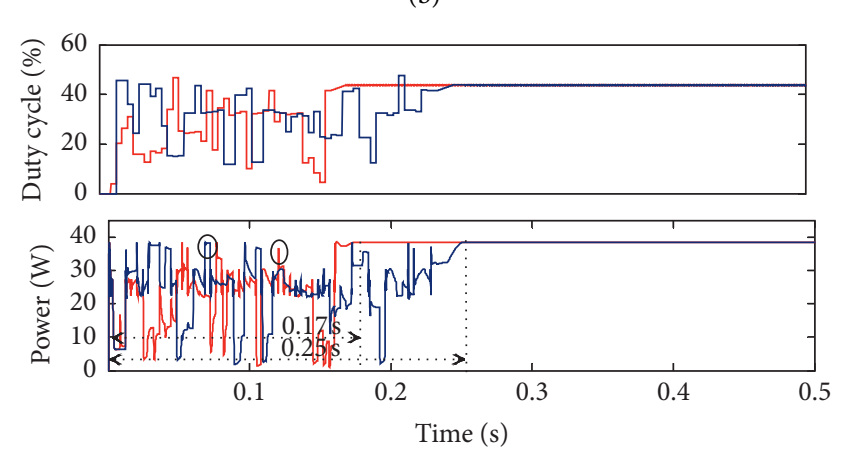

_ Variable Ts

— Fixed Ts

(c)

(d)

FIGURE 8: The simulation results of the proposed method under different shading patterns. (a) The shading pattern 1, (b) the shading pattern 2, (c) the shading pattern 3 , and (d) the shading pattern 4 . 
TABLE 1: Comparison results of the proposed method with previous works.

\begin{tabular}{lccc}
\hline GMPPT algorithm & PV power $(\mathrm{W})$ & Tracking speed (S) & Steady-state efficiency (\%) \\
\hline P\&O [25] & 34.8 & 1.38 & 79.6 \\
Fixed-step INC [26] & 148.5 & 0.55 & 7.88 \\
PSO [25] & 34.8 & 3.15 & 100 \\
Combined PSO and P\&O [25] & 30.6 & 2.94 & 99.9 \\
Random search [27] & 22.2 & 2.82 & 100 \\
Continuous GA [28] & 40502.4 & 1.96 & 100 \\
Flashing fireflies [29] & 66.6 & 1.20 & 99.9 \\
Cuckoo search [30] & 87.5 & 0.88 & 99.9 \\
Improved cuckoo search [30] & 87.5 & 0.47 & 99.9 \\
Flower pollination [31] & 113.1 & 0.42 & 9.9 .8 \\
Fuzzy logic [26] & 157.3 & 0.18 & 98 \\
The proposed method fixed $T_{\mathrm{s}}$-pattern 1 & 48.8 & 0.24 & 100 \\
The proposed method variable $T_{\mathrm{s}}$-pattern 1 & 48.8 & 0.16 & 100 \\
The proposed method fixed $T_{\mathrm{s}}$-pattern 2 & 60.3 & 0.27 & 100 \\
The proposed method variable $T_{\mathrm{s}}$-pattern 2 & 60.3 & 0.19 & 100 \\
The proposed method fixed $T_{\mathrm{s}}$-pattern 3 & 32.2 & 0.27 & 100 \\
The proposed method variable $T_{\mathrm{s}}$-pattern 3 & 32.2 & 0.17 & 100 \\
The proposed method fixed $T_{\mathrm{s}}$-pattern 4 & 38.4 & 0.25 & 100 \\
The proposed method variable $T_{\mathrm{s}}$-pattern 4 & 38.4 & 100 \\
\hline
\end{tabular}

satisfied, $\mathrm{P} \& \mathrm{O}$ algorithm runs to track GMPP in response to irradiance and temperature changes. Furthermore, the stopping criterion of FWA can be further relaxed to increase the tracking speed. In fact, FWA is stopped if the stopping criterion is satisfied four times. However, FWA finds GMPP much sooner than the stopping criterion, according to Figure 8, so the tracking speed can be further increased by relaxing the stopping criterion.

5.2. Discussion and Comparison. To prove the superiority of the proposed method, different types of MPPT and GMPPT algorithms are surveyed and compared in $\mathrm{Ta}$ ble 1 . To this end, the surveyed methods are evaluated based on the tracking speed and the steady-state efficiency. According to Table 1, conventional methods like $\mathrm{P} \& \mathrm{O}$ or INC have either fast tracking speed or high steady-state efficiency. Therefore, there is a trade-off between the tracking speed and the steady-state efficiency as well as getting trapped in local MPPs. Particle Swarm Optimization (PSO) reaches high efficiency in the steady state; however, it has a sluggish dynamic behaviour. Therefore, it is combined with $\mathrm{P} \& \mathrm{O}$ to address this problem. Among the other smart methods, flower pollination and improved cuckoo search have high tracking speed. Regarding the proposed method, its results show that it has the highest tracking speed in different shading patterns and maintains high steady-state efficiency simultaneously.

\section{Experimental Results}

An experimental laboratory setup was built to verify the theoretical analysis of the proposed algorithm. The algorithm is implemented on Texas Instruments TMS320F2812 Digital Signal Processing (DSP) platform. To this end, the Event Manager unit is configured to

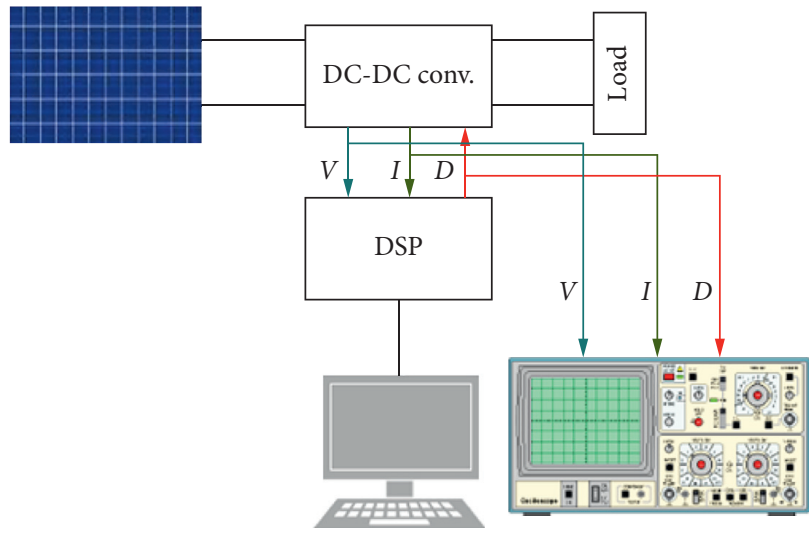

FIgURE 9: Block diagram of the experimental setup.

TABLE 2: Electrical parameters of the PV panel.

\begin{tabular}{lc}
\hline PV panel parameters & Nominal values $(\mathrm{W})$ \\
\hline$P_{\max }$ & 60 \\
VMPP & 18.5 \\
IMPP & 3.25 \\
VOC & 22.9 \\
ISC & 3.44 \\
\hline
\end{tabular}

produce $60 \mathrm{kHz}$ Pulse Width Modulation (PWM) signal, and consequently it is passed through the optocoupler HCPL 3120 to isolate DSP from the boost converter. Moreover, the PV voltage and current are sampled at 60 $\mathrm{kHz}$ frequency. Block diagram of the experimental setup is shown in Figure 9.

Two YL60P-17b PV panels, whose electrical parameters are mentioned in Table 2, are connected in series, and since each of them has two bypass diodes, the whole string can be treated as a string constituting of four 30-watt PV panels in series. Therefore, it is possible to create four MPPs at the 


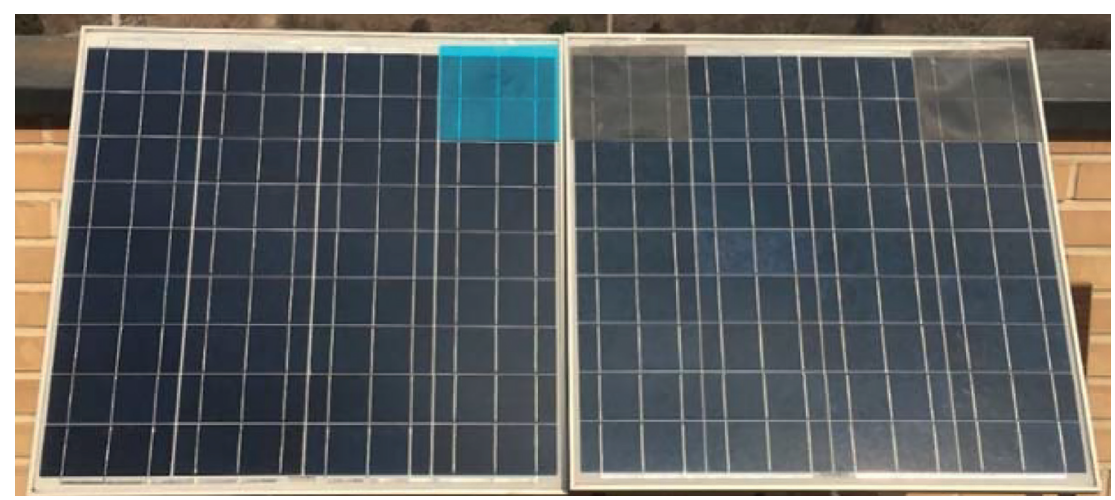

(a)

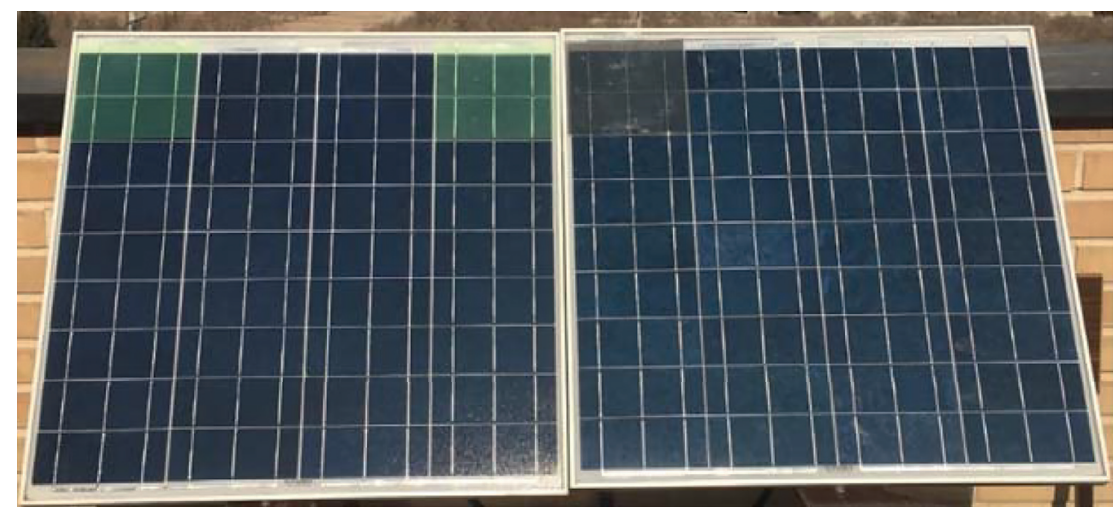

(b)

FIGURE 10: Different partial shading patterns created by coloured glasses. (a) Shading pattern 1. (b) Shading pattern 2.

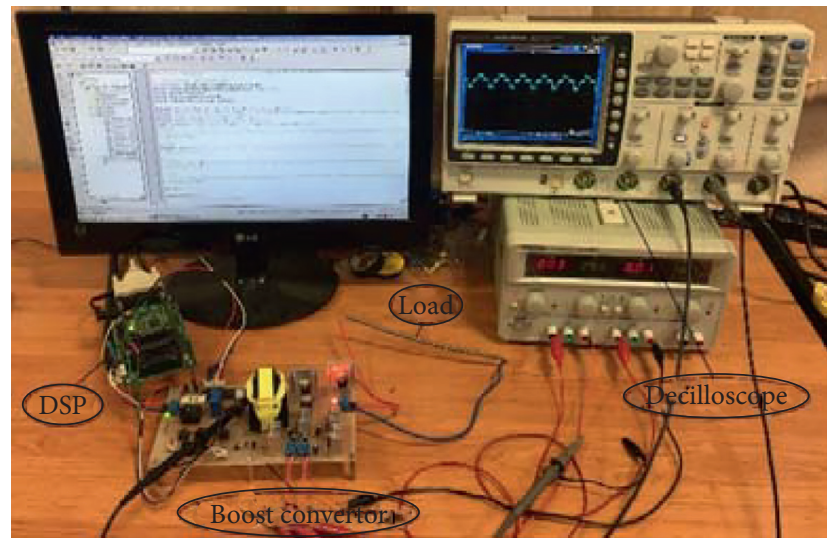

FIGURE 11: The laboratory prototype of the proposed system.

most by placing colourful glasses on different parts of the PV panels. The photos of the PV panels to prepare the shading patterns are shown in Figure 10. Also, the laboratory prototype photo of the proposed system is shown in Figure 11.

Finally, Code Composer Studio (CCS) software which has real-time interaction with DSP platform is used to investigate the $\mathrm{PV}$ power. Therefore, the $\mathrm{PV}$ power is captured in CCS and is shown in Figures 12 and 13 where
FWA successfully finds GMPP in different shading patterns.

Figure 12 shows the PV power convergence to GMPP by the proposed method for the shading pattern 1. In Figure 12(a), the PV power in the fixed sampling situation is shown. Also, in Figure 12(b), the PV power in the variable sampling situation is shown. As it can be seen from this figure, the PV power in variable sampling time has reached to its final value faster. 


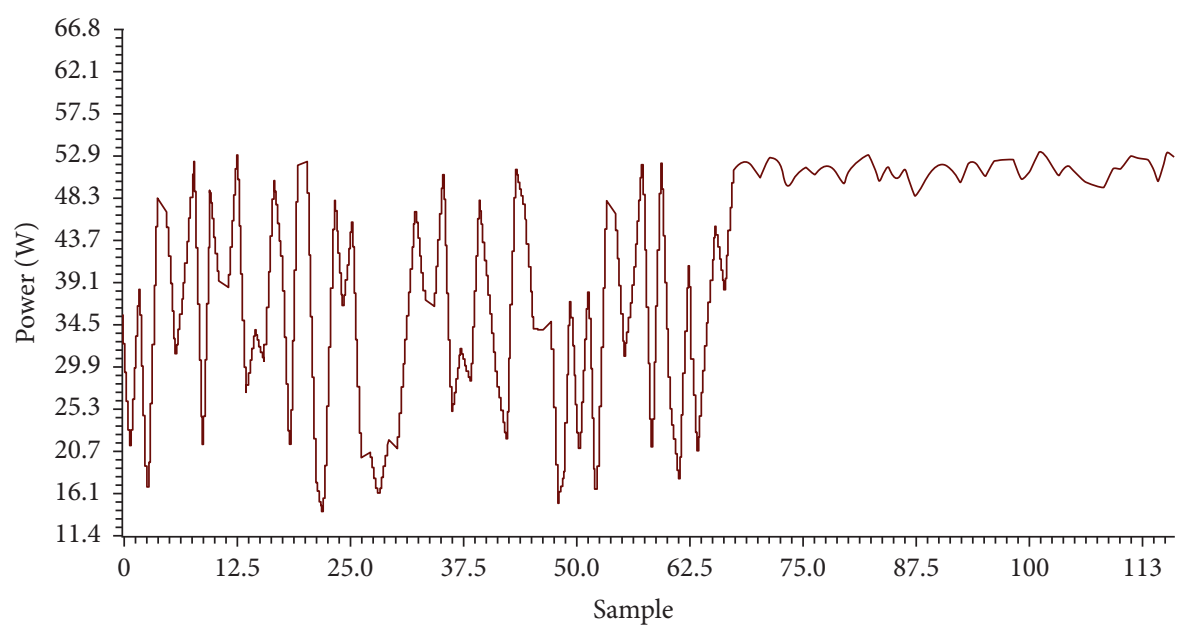

(a)

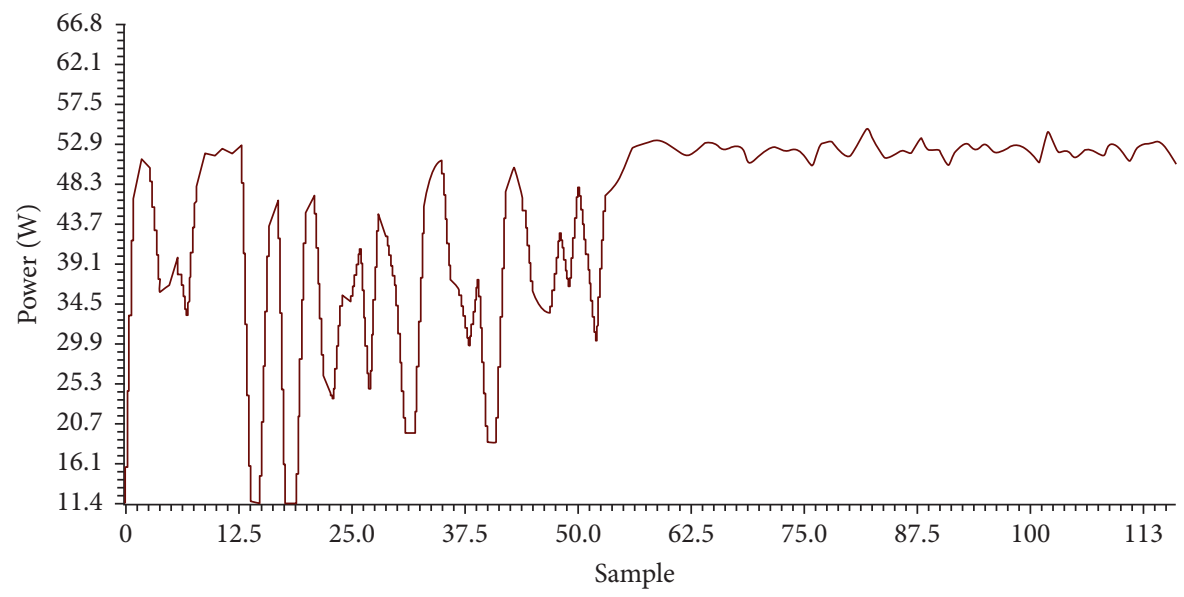

(b)

Figure 12: The PV power convergence to GMPP by the proposed method for the shading pattern 1. (a) With the fixed sampling time technique. (b) With the variable sampling time technique.

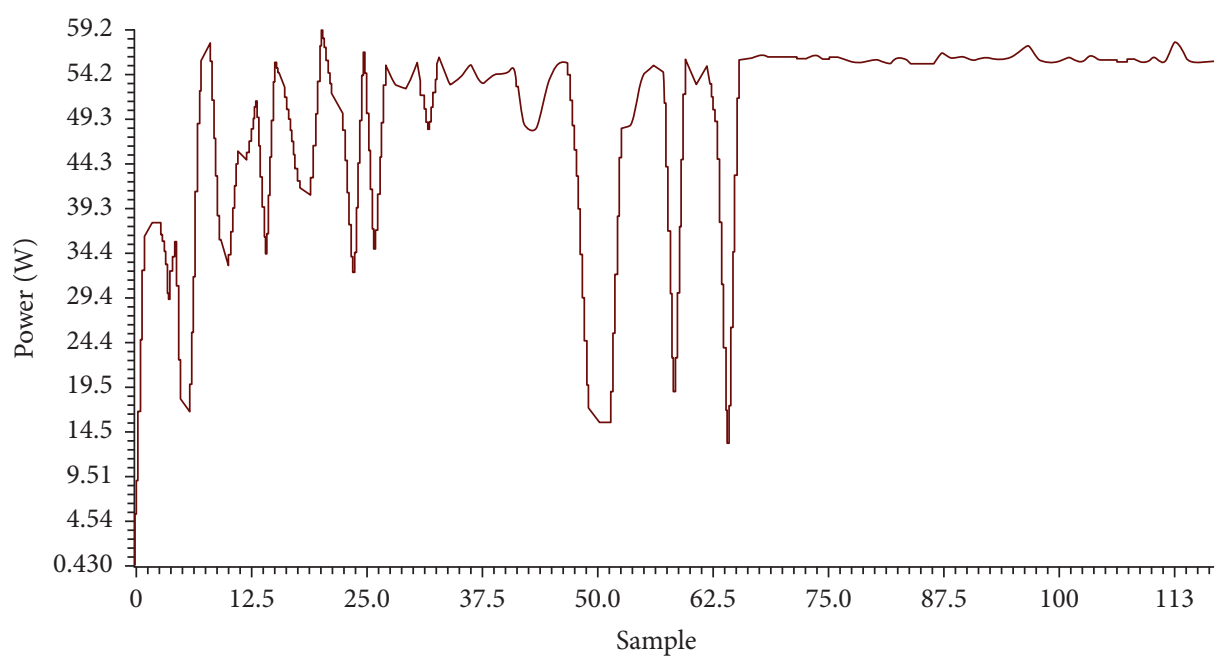

(a)

Figure 13: Continued. 


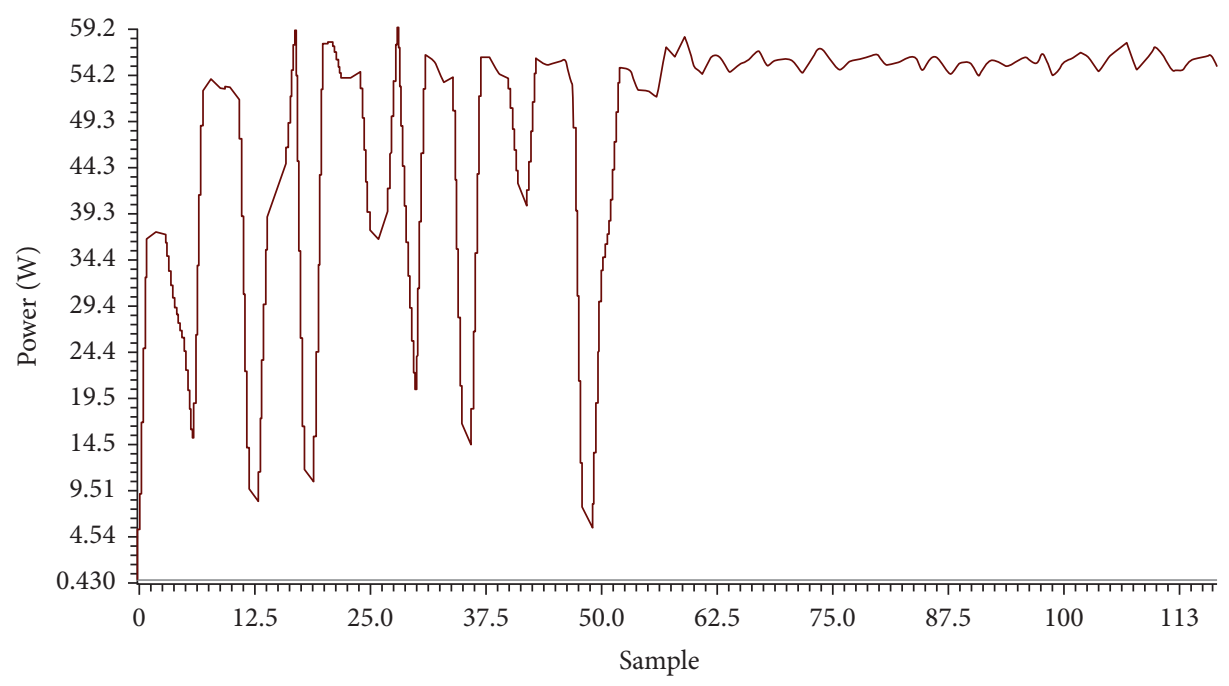

(b)

FIgURE 13: The PV power convergence to GMPP by the proposed method for the shading pattern 2. (a) With the fixed sampling time technique. (b) With the variable sampling time technique.

Figure 13 shows the experimental results for the shading pattern 2. The PV power curves in the fixed and variable sampling situations are shown in Figures 13(a) and 13(b), respectively. In this pattern, the changes in PV power in variable sampling time have more speed.

\section{Conclusion}

The nonlinear characteristic of PV systems has become more complicated when they are partially shaded. Therefore, advanced GMPPT techniques are required to maximize the PV system efficiency. In this paper, a novel GMPPT algorithm is proposed to tackle obstacles which stem from PSCs. The novelty of the proposed method can be summarized in three parts. Firstly, it has high tracking speed compared with previous works. Secondly, the computational effort is reduced by combining FWA with $\mathrm{P} \& \mathrm{O}$ so that FWA is only used when PSC occurs; afterwards, the simple $\mathrm{P} \& \mathrm{O}$ is run. Thirdly, based on the nature of FWA which can spread the fireworks and sparks in a wide area with different radii, the proposed method can find GMPP in complicated shading patterns. Finally, based on the comparison in Table 1, the simulation and experimental results, the reliability of the proposed method is proven.

\section{Data Availability}

No data were used to support this study.

\section{Conflicts of Interest}

The authors declare that there are no conflicts of interest regarding the publication of this paper.

\section{References}

[1] M. H. Boujmil, A. Badis, and M. N. Mansouri, "Nonlinear robust backstepping control for three-phase grid-connected pv systems," Mathematical Problems in Engineering, vol. 2018, Article ID 3824628, 13 pages, 2018.

[2] M. A. Husain, A. Jain, A. Tariq, and A. Iqbal, "Fast and precise global maximum power point tracking techniques for photovoltaic system," IET Renewable Power Generation, vol. 13, no. 14, pp. 2569-2579, 2019.

[3] X. Li, H. Wen, Y. Hu, and L. Jiang, "A novel beta parameter based fuzzy-logic controller for photovoltaic MPPT application," Renewable Energy, vol. 130, pp. 416427, 2019.

[4] E. M. Vicente, P. dos Santos Vicente, R. L. Moreno, and E. R. Ribeiro, "High-efficiency MPPT method based on irradiance and temperature measurements," IET Renewable Power Generation, vol. 14, no. 6, pp. 986-995, 2020.

[5] P.-C. Chen, P.-Y. Chen, Y.-H. Liu, J.-H. Chen, and Y.-F. Luo, "A comparative study on maximum power point tracking techniques for photovoltaic generation systems operating under fast changing environments," Solar Energy, vol. 119, pp. 261-276, 2015.

[6] J. Ahmed and Z. Salam, "An enhanced adaptive P\&O MPPT for fast and efficient tracking under varying environmental conditions," IEEE Transactions on Sustainable Energy, vol. 9, no. 3, pp. 1487-1496, 2018.

[7] V. R. Kota and M. N. Bhukya, "A novel global MPP tracking scheme based on shading pattern identification using artificial neural networks for photovoltaic power generation during partial shaded condition," IET Renewable Power Generation, vol. 13, no. 10, pp. 1647-1659, 2019.

[8] T. Sudhakar Babu, K. Sangeetha, and N. Rajasekar, "Voltage band based improved particle swarm optimization technique for maximum power point tracking in solar photovoltaic system," Journal of Renewable and Sustainable Energy, vol. 8, no. 1, Article ID 013106, 2016. 
[9] L. Chen and X. Wang, "Enhanced MPPT method based on ANN-assisted sequential Monte-Carlo and quickest change detection," IET Smart Grid, vol. 2, no. 4, pp. 635-644, 2019.

[10] H. Patel and V. Agarwal, "Maximum power point tracking scheme for PV systems operating under partially shaded conditions," IEEE Transactions on Industrial Electronics, vol. 55, no. 4, pp. 1689-1698, 2008.

[11] H. Renaudineau, A. Houari, J.-P. Martin, S. Pierfederici, F. Meibody-Tabar, and B. Gerardin, "A new approach in tracking maximum power under partially shaded conditions with consideration of converter losses," Solar Energy, vol. 85, no. 11, pp. 2580-2588, 2011.

[12] X. Li, H. Wen, Y. Hu, L. Jiang, and W. Xiao, "Modified beta algorithm for GMPPT and partial shading detection in photovoltaic systems," IEEE Transactions on Power Electronics, vol. 33, no. 3, pp. 2172-2186, 2018.

[13] M. E. Başoğlu, "An improved 0.8 VOC model based GMPPT technique for module level photovoltaic power optimizers," IEEE Transactions on Industry Applications, vol. 55, no. 2, pp. 1913-1921, 2019.

[14] R. Motamarri and B. Nagu, "GMPPT by using PSO based on Lévy flight for photovoltaic system under partial shading conditions," IET Renewable Power Generation, vol. 14, no. 7, pp. 1143-1155, 2020.

[15] T. L. Nguyen and K.-S. Low, "A global maximum power point tracking scheme employing direct search algorithm for photovoltaic systems," IEEE Transactions on Industrial Electronics, vol. 57, no. 10, pp. 3456-3467, 2010.

[16] G. Carannante, C. Fraddanno, M. Pagano, and L. Piegari, "Experimental performance of MPPT algorithm for photovoltaic sources subject to inhomogeneous insolation," IEEE Transactions on Industrial Electronics, vol. 56, no. 11, pp. 4374-4380, 2009.

[17] K. L. Lian, J. H. Jhang, and I. S. Tian, “A maximum power point tracking method based on perturb-and-observe combined with particle swarm optimization," IEEE Journal of Photovoltaics, vol. 4, no. 2, pp. 626-633, 2014.

[18] N. Pragallapati, T. Sen, and V. Agarwal, "Adaptive velocity PSO for global maximum power control of a PV array under nonuniform irradiation conditions," IEEE Journal of Photovoltaics, vol. 7, no. 2, pp. 624-639, 2016.

[19] C. Manickam, G. P. Raman, G. R. Raman, S. I. Ganesan, and N. Chilakapati, "Fireworks enriched P\&O algorithm for GMPPT and detection of partial shading in PV systems," IEEE Transactions on Power Electronics, vol. 32, no. 6, pp. 44324443, 2017.

[20] S. Zheng, A. Janecek, and Y. Tan, "Enhanced fireworks algorithm," in Proceedings of the 2013 IEEE Congress on Evolutionary Computation (IEEE), pp. 2069-2077, Cancun, Mexico, June 2013.

[21] B. Zhang, Y.-J. Zheng, M.-X. Zhang, and S.-Y. Chen, "Fireworks algorithm with enhanced fireworks interaction," IEEE/ ACM Transactions on Computational Biology and Bioinformatics, vol. 14, no. 1, pp. 42-55, 2015.

[22] S. Zheng, J. Li, A. Janecek, and Y. Tan, "A cooperative framework for fireworks algorithm," IEEE/ACM Transactions on Computational Biology and Bioinformatics, vol. 14, no. 1, pp. 27-41, 2015.

[23] N. Priyadarshi, S. Padmanaban, J. B. Holm-Nielsen, F. Blaabjerg, and M. S. Bhaskar, "An experimental estimation of hybrid ANFIS-PSO-based MPPT for PV grid integration under fluctuating sun irradiance," IEEE Systems Journal, vol. 14, no. 1, pp. 1218-1229, 2020.
[24] M. Mao, L. Zhou, Z. Yang et al., "A hybrid intelligent GMPPT algorithm for partial shading PV system," Control Engineering Practice, vol. 83, pp. 108-115, 2019.

[25] K. Zhang, V. Vignesh kumar, and S. Palani, “Application of a combined particle swarm optimization and perturb and observe method for MPPT in PV systems under partial shading conditions," Renewable Energy, vol. 75, pp. 308-317, 2015.

[26] Y.-T. Chen, Y.-C. Jhang, and R.-H. Liang, "A fuzzy-logic based auto-scaling variable step-size MPPT method for PV systems," Solar Energy, vol. 126, pp. 53-63, 2016.

[27] K. Sundareswaran, S. Palani, and S. Peddapati, "Application of random search method for maximum power point tracking in partially shaded photovoltaic systems," IET Renewable Power Generation, vol. 8, no. 6, pp. 670-678, 2014.

[28] G. Shankar and V. Mukherjee, "MPP detection of a partially shaded PV array by continuous GA and hybrid PSO," Ain Shams Engineering Journal, vol. 6, no. 2, pp. 471-479, 2015.

[29] K. Sundareswaran, S. Peddapati, and S. Palani, "MPPT of PV systems under partial shaded conditions through a colony of flashing fireflies," IEEE Transactions on Energy Conversion, vol. 29, no. 2, pp. 463-472, 2014.

[30] J.-Y. Shi, F. Xue, Z.-J. Qin, W. Zhang, L.-T. Ling, and T. Yang, "Improved global maximum power point tracking for photovoltaic system via cuckoo search under partial shaded conditions," Journal of Power Electronics, vol. 16, no. 1, pp. 287-296, 2016.

[31] J. P. Ram and N. Rajasekar, "A new global maximum power point tracking technique for solar photovoltaic (PV) system under partial shading conditions (PSC)," Energy, vol. 118, pp. 512-525, 2017. 